\title{
Esclerosis Lateral Amiotrófica, presentación atípica
}

\author{
Amparo Manzano Juárez ${ }^{a}$, María Dolores González Céspedes ${ }^{b}$, Eddy Rocha Honor ${ }^{c}$ y \\ María Pilar Sánchez Beteta ${ }^{d}$
}

aM.I.R. Medicina de Familia y Comunitaria. Centro de Salud Hellín II. Albacete (España). 'Médico de Familia. Centro de Salud Hellín II. Albacete (España)

'Médico de Familia. Centro de Rehabilitación Geronto Geriátrico Integral S.R.L. de Cochabamba. (Bolivia). 'Médico de Familia. Centro de Salud de Tobarra. Albacete (España).

Correspondencia: Amparo Manzano Juárez. C/ Jacinto Benavente 19, $4^{\circ}$ V. C.P. 02400 . Hellín (Albacete). España.

Correo electrónico: ampamanjua@hotmail.com.

Recibido el 15 de marzo de 2015.

Aceptado para su publicación el 24 de abril de 2015.

\section{RESUMEN}

La esclerosis lateral amiotrófica (ELA) es una enfermedad neurodegenerativa, progresiva, con desenlace fatal, que afecta a neuronas motoras de la médula espinal, tronco cerebral y corteza motora. Se produce un fracaso del sistema motor que dirige, regula y mantiene la musculatura esquelética, responsable de la capacidad para moverse y relacionarse con el entorno. Presentamos el caso de un hombre de 58 años de edad con diagnóstico de ELA, con sintomatología inicial atípica que dificultó el juicio clínico final, resaltando en el diagnóstico diferencial la miopatía por cuerpos de inclusión.

PALABRAS CLAVE: Esclerosis Amiotrófica Lateral. Enfermedad de la Neurona Motora. Miositis por Cuerpos de Inclusión.

\section{ABSTRACT}

\section{Atypical presentation of Amyotrophic lateral sclerosis}

Amyotrophic lateral sclerosis (ALS) is a progressive, neurodegenerative fatal disease that affects motor neurons in the spinal cord, brain stem and motor cortex. It produces a failure of the motor system that directs, adjusts and maintains skeletal muscles, responsible for the ability to move and interact with the environment. We report the case of a 58-year-old male diagnosed with ALS, with atypical initial symptoms that hindered the final clinical judgement, and where inclusion body myopathy stood out during the differential diagnosis.

KEY WORDS: Amyotrophic Lateral Sclerosis. Motor Neuron Disease. Myositis, Inclusion Body.

\section{INTRODUCCIÓN}

La esclerosis lateral amiotrófica (ELA) afecta principalmente a adultos de entre 40 y 70 años. El inicio es a los 60,5 años en las formas no familiares, y 10 años antes en las familiares. La proporción entre hombres y mujeres es de 1,3/1. La incidencia en la población española es de 1-3/100.000 y la prevalencia es de 3,5/100.000. Existen focos endémicos con mayor prevalencia en regiones del Pacífico Occidental con una incidencia de 50-150 veces superior; en cambio, es menor en la población africana, asiática e hispánica. La mayoría de los pacientes con ELA fallecen por fallo respiratorio, generalmente entre 3 y 5 años desde el inicio de los síntomas ${ }^{1-4}$.

La miopatía por cuerpos de inclusión en una forma de miopatía inflamatoria sistémica poco frecuente, con una prevalencia de 8 casos por 100.000 habitantes. La etiopatogenia es desconocida, pero existen datos que apuntan a la naturaleza autoinmune. Afecta de forma asimétrica tanto a la musculatura proximal (70 \% de los casos) como distal (20\% de los casos), especialmente al cuádriceps y a los flexores de la muñeca, dedos y tobillo, lo que provoca caídas frecuentes. La disfagia aparece en el $40 \%$ de los casos. El diagnóstico es clínico e histopatológico. Es más común en varones (60-70 \% de los casos) de más de 50 años, y su desarrollo y progresión es lento (meses a años) $)^{5-8}$. 


\section{CASO CLÍNICO}

Paciente de 59 años que refiere dificultad para caminar desde hace un mes aproximadamente. Presenta debilidad y dolor en los miembros inferiores, que mejora tras detenerse, acompañándose en ocasiones de lumbalgia no irradiada. Ha notado cambios en la voz, siendo ésta más disfónica, que relaciona con su trabajo. En la anamnesis dirigida no refiere debilidad en las manos o extremidades superiores. No presenta trastorno ocular, ni de control de esfínteres y tampoco a nivel sensitivo. No se aprecia labilidad emocional, aunque el paciente ya presentaba un trastorno adaptativo tras la muerte de su mujer. Refiere calambres ocasionales en gemelos. Como antecedentes personales, es hipertenso y diabético tipo 2. Antecedentes familiares: tío paterno fallecido con el diagnóstico de Esclerosis lateral amiotrófica (ELA).

En la exploración neurológica presenta habla hipofónica, facial centrado. Motilidad ocular interna y externa normal. Fasciculaciones linguales. No debilidad cervical. Motor: miembros inferiores (MMII): paraparesia de predominio proximal 4/5. Dorsiflexión de ambos pies $5 / 5$. No se objetiva debilidad en miembros superiores. No déficit sensitivo. Reflejo osteo-tendinoso (ROT) +++/+++ con Hoffmann positivo bilateral y reflejo cutáneo-plantar derecho indiferente, izquierdo conservado. Fasciculaciones en deltoides, pectorales y de forma difusa en MMII. Atrofia muscular en ambos cuádriceps. Exploración general anodina.

Se realiza índice tobillo-brazo (ITB): derecho 1,18; izquierdo 1,29. Se solicita analítica, donde destaca CK de 232, estudio inmunológico y serología normal. Radiografía de tórax y lumbosacra normal. Tras sospecha de enfermedad de motoneurona se interconsulta al servicio de Medicina Interna, que solicita electromiograma (EMG) / electroneurografía (ENG) sugestivo de patrón neurógeno con denervación activa en ambos MMII, miembro superior derecho (fibrilaciones y ondas positivas), compatible con enfermedad de motoneurona, probable ELA. Con estos resultados se derivó a Neurología.

Tras la valoración del paciente y con diagnóstico de probable ELA, según los criterios revisados de El Escorial, y pendiente de completar estudio con resonancia magnética craneal-medular y nuevo estudio neurolofisiológico, se inició tratamiento con Riluzol 50 mg, un comprimido cada 12 horas.

Debido a la evolución clínica del paciente con paraparesia proximal en MMII, con importante atrofia muscular de ambos muslos, disfagia para sólidos, sin afectación en miembros superiores (MMSS) y correlacionado con último estudio neurofisiológico, se planteó otro posible diagnóstico, en concreto una miopatía. EI EMG sugiere miopatía con afectación bulbar y de cintura pelviana (miopatía por cuerpos de inclusión), por lo que se realizó biopsia muscular, siendo los resultados de la misma contundentes, descartando la posibilidad de enfermedad primaria del musculo esquelético y evidenciándose únicamente cambios secundarios a denervación.

El paciente posteriormente presentó progresión de los síntomas, evidenciándose debilidad en la flexión dorsal del pie izquierdo (4/5). Atrofia incipiente a nivel del quinto espacio interóseo de la mano izquierda. Fasciculaciones difusas por diferentes segmentos corporales, así como leve afonía.

Finalmente se sugiere el diagnóstico de enfermedad de motoneurona, probable ELA. Se continúa tratamiento con Riluzol $50 \mathrm{mg}$. Se recomienda logopedia y rehabilitación domiciliaria física y respiratoria.

Tras 5 meses del diagnóstico definitivo el paciente presenta un shock séptico de origen urinario y siguientes complicaciones, precisando ventilación mecánica invasiva (traqueotomía), alimentación por gastrostomía percutánea (PEG) y trastorno adaptativo secundario, dándose de alta al domicilio. A los dos meses presenta exitus.

\section{DISCUSIÓN}

La ELA debuta con la combinación de signos y síntomas de la degeneración de la primera o segunda motoneurona. Puede presentarse con afectación focal o multifocal con posterior progresión a otras regiones. Los primeros síntomas de la enfermedad se manifiestan por la aparición de debilidad y pérdida de fuerza localizada en un grupo muscular en cualquiera de las extremidades. Esta sintomatología inespecífica tiene como consecuencia un retraso en el diagnóstico, de ahí la importancia que el médico de familia conozca esta clínica y la tenga en cuenta en el diagnóstico diferencial. En nuestro caso el paciente debutó con debilidad de miembros inferiores y síntomas sugestivos de claudicación intermitente, descartándose por ITB. En la exploración física se observó la presencia de fasciculaciones en distintos grupos musculares y atrofia de cuádriceps, signos guía para el inicio de estudio de enfermedad de motoneurona. Es característico de la ELA la afectación precoz de los miembros 
superiores con atrofia de los músculos interóseos, que posteriormente se extiende a los extensores del antebrazo y a zonas más proximales, acompañándose de fasciculaciones. Esto fue lo que creó confusión en nuestro caso, con clínica de debilidad muscular distal. A nivel de miembros inferiores esta enfermedad se manifiesta en el cuádriceps y en los músculos de cara antero-externa de las piernas. El compromiso bulbar se presenta como paresia progresiva de la deglución, disartria e hipofonía. No existe compromiso de esfínteres ni de la musculatura ocular extrínseca hasta estadios avanzados, y las funciones autonómicas suelen mantenerse intactas aun en fases finales de la enfermedad.

La evolución de la enfermedad es irreversible, llevando al paciente a la inmovilidad casi completa, con severos trastornos deglutorios e insuficiencia respiratoria restrictiva por debilidad de los músculos intercostales y del diafragma, factores que son determinantes en la muerte de estos pacientes.

El diagnóstico de esta patología es esencialmente clínico, reflejado en los criterios de El Escorial revisados en 1998, aunque posteriormente se establecen los criterios de Arlie, en los que se añaden criterios electromiográficos y de imagen.

Respecto al tratamiento etiológico no existe ninguno de eficacia probada. El Riluzol (50 mg/12h) es por el momento el único fármaco aprobado que logra retrasar su evolución, especialmente en pacientes con inicio bulbar, aplazando la necesidad de ventilación mecánica. La fisioterapia, la rehabilitación y el uso de ayudas mecánicas pueden mejorar la función muscular y contribuir al bienestar de estos pacientes. Esta enfermedad genera un importante cambio en la estructura y dinámica familiar con problemas emocionales, psicológicos y económicos que requieren respuestas ágiles, coordinadas y accesibles para el enfermo y el entorno familiar. Al ser una enfermedad degenerativa e invalidante es de vital importancia el seguimiento y coordinación por parte del equipo de Atención Primaria $^{1-4,9-11}$.

\section{BIBLIOGRAFÍA}

1. Raventós Galcerán M, Ribas Batllori MA, Khouli M. Esclerosis lateral amiotrófica. Una enfermedad de difícil diagnóstico. A propósito de dos casos. FMC. 2011; 18(5):2558.

2. Maragakis NJ, Gálvez-Jiménez N. Epidemiology and pathogenesis of amyotrophic lateral sclerosis [Monografía en Internet]. UpToDate [Actualizado 30/01/2015; Acceso 17/04/2015]. Disponible en: http://www.uptodate.com

3. Grupo de trabajo de la Guía para la Atención de la Esclerosis Lateral Amiotrófica (ELA) en España. Guía para la Atención de la Esclerosis Lateral Amiotrófica (ELA) en España. Madrid: Ministerio de Sanidad y Política Social; 2009.

4. García Gálvez P, Sebastián Cambón R, Higes Pascual F, Sánchez-Migallón MJ, Yusta Izquierdo A. Enfermedad de la motoneurona. Medicine. 2011; 10 (77): 5200-8.

5. Miller ML. Clinical manifestations and diagnosis of inclusion body myositis [Monografía en Internet]. UpToDate [Actualizado 11/11/2014; Acceso 17/04/2015]. Disponible en: http://www.uptodate.com

6. Turrión Nieves Al, Martín Holguera R, Sánchez Atrio Al, Moruno Cruz H. Miopatías inflamatorias idiopáticas. Medicine. 2013; 11 (33): 2040-7.

7. Selva O'Callaghan A, Trallero Araguás E. Miopatías inflamatorias. Dermatomiositis, polimiositis y miositis con cuerpos de inclusión. Reumatol Clin. 2008; 4 (5): 197-206.

8. Selva-O'Callaghan A, Grau-Junyent JM, Trallero-Araguás E, Labrador-Horrillo M. Miopatías idiopáticas inflamatorias. Medicine. 2009; 10 (31): 2073-82.

9. Elman LB, McCluskey L. Clinical features of amyotrophic lateral sclerosis and other forms of motor neuron disease [Monografía en Internet]. UpToDate [Actualizado 28/07/2014; Acceso 17/04/2015]. Disponible en: http:// www.uptodate.com

10. Quarracino C, Rey RC, Rodríguez GE. Esclerosis lateral amiotrófica (ELA): seguimiento y tratamiento. Neurol Arg. 2014; 6 (2): 91-5.

11. Choudry RB, Gálvez-Jiménez N, Cudkowicz ME. Disease modifying treatment of amyotrophic lateral sclerosis [Monografía en Internet]. UpToDate [Actualizado 18/03/2015; Acceso 17/04/2015]. Disponible en: http://www.uptadate. com 